\title{
ANÁLISE DE EXATIDÃO POSICIONAL DE ORTOMOSAICOS GERADOS A PARTIR DE DADOS TERMAIS A BORDO DE VANT
}

\author{
Accuracy analysis of ortho-mosaic produced from a thermal sensor aboard UAV
}

\section{Hermann Johann Heinrich Kux ${ }^{1}$, Luana Thayza de Oliveira ${ }^{2}$, Fabiano da Cruz Nogueira ${ }^{3}$}

${ }^{12}$ INPE-Divisão de Sensoriamento Remoto, Caixa postal 515 - 12227-010 - São José dos Campos - SP, Brasil e luana.oliveira@inpe.br, hermann.kux @inpe.br, ${ }^{3}$ IEAv-Divisão ECR-S, Tr. Cel Av José Alberto A. do Amarante, 01 São José dos Campos - SP, Brasil 12228-001 e nogueirafcn@fab.mil.br

\section{RESUMO}

O objetivo deste trabalho foi avaliar a exatidão posicional dos produtos resultantes do uso de um sensor termal em um VANT, ortomosaico termal, segundo o Padrão de Exatidão Cartográfica dos Produtos Cartográficos Digitais (PEC-PCD). Para tanto, foi realizado um levantamento de dados termais utilizando um VANT multi-rotor quadricóptero numa área de aproximadamente $10.000 \mathrm{~m}^{2}$ em São José dos Campos, SP. Duas configurações de processamento foram utilizadas para a geração dos produtos: uma utilizando apenas as coordenadas centrais das imagens e outra, com a adoção de pontos de controle no processamento.

Desde que vinculado a utilização de pontos de apoio para o processamento e consideradas as incertezas envolvidas no seu processo de obtenção, constatou-se a viabilidade do uso de dados termais obtidos por VANT como uma alternativa eficaz para imageamento de áreas com características não aparentes na faixa do visível, ou em períodos noturnos.

Palavras-chave: Infravermelho Termal, VANT, Exatidão Posicional.

\section{ABSTRACT}

The objective of this study was to evaluate the positional accuracy of products from the use of a thermal sensor, a thermal ortho-mosaic, in accordance with the Brazilian Cartographic Accuracy Standard of Digital Cartographic Products (PEC-PCD). In order to perform these tasks, a field survey with thermal data was done, using a multirotor quadri-copter drone, at an area of approximately $10,000 \mathrm{~m}^{2}$, in São José dos Campos, SP. Two processing configurations were used to generate the products: 1 . using only the central coordinates of the images and 2 . considering Ground Control points during processing. Taking into account the mandatory use of GCPs and the uncertainties involved in the process to obtain thermal images with a drone, this is an efficient alternative to image areas with non-apparent characteristics in the visible spectrum or at night.

Keywords: Thermal Infrared. UAV, Characterization. Position accuracy.

\section{INTRODUÇÃO}

O Sensoriamento Remoto Termal é um ramo do Sensoriamento Remoto que opera com a aquisição, processamento e interpretação dos dados adquiridos na faixa do infravermelho termal do espectro eletromagnético. A detecção termal é utilizada para a exploração geológica, detecção de incêndios, agricultura, etc.

Contudo, a larga utilização destes dados pela sociedade civil esbarra em alguns desafios. Dentre os sistemas TIR existentes precisa ser ainda considerada a sua limitação quanto à frequência de imageamento e resolução espacial.

Alternativamente, sensores termais podem ser utilizados em aeronaves, o que resulta em um ganho em termos de resolução espacial e na flexibilidade temporal da coleta de dados. No entanto, [1] afirma que o imageamento termal aéreo não tem sido largamente disponível ao público geral porque tais missões envolvem custos relativamente altos.
Atualmente, este cenário está sendo alterado. Um dos motivos é o uso de Veículos Aéreos Não Tripulados (VANTs), de custo reduzido em comparação com outras plataformas para obtenção de dados [2]. Aliado ao aumento da disponibilidade de veículos aéreos não tripulados de baixo custo, os sensores termais, sobretudo os sensores não refrigerados, vêm sendo desenvolvidos com o intuito de adaptação em plataformas não tripuladas.

Essas mudanças recentes têm estimulado o maior uso de sensores que operam nesta faixa espectral a bordo de veículos aéreos não tripulados, com diversas aplicações, a saber: áreas, vide [3], [2], [4], [5], [6], [7] e [8]. Neste contexto, o objetivo deste trabalho foi avaliar a exatidão posicional dos produtos resultantes de levantamento de dados termais utilizando um VANT, segundo o Padrão de Exatidão Cartográfica (PECPCD).

\section{MATERIAL E MÉTODOS}

\subsection{Sistema sensor}

O sistema sensor utilizado para a obtenção das imagens foi um Kit PI LightWeight que compreende um microprocessador e um sensor [9] OPTRIS PI 450. Este possui características específicas, o que permite embarcá-lo em VANTs. As especificações técnicas do sistema sensor estão apresentadas na Tabela 1.

Tabela 1. Especificações técnicas dos sensor calibrado

\begin{tabular}{lc}
\hline Dados de imageamento & Sensor \\
\hline Número de pixels & $382 \times 288$ \\
Campo de visada (FOV) & $62^{\circ} \times 49^{\circ}$ \\
Distância focal & $8 \mathrm{~mm}$ \\
Resolução espacial (IFOV) & $1,81 \mathrm{mrad}$ \\
Sensibilidade térmica/NETD & $0,04 \mathrm{~K}$ \\
Frequência & $80 \mathrm{~Hz}$ \\
Dados do detector & \\
\hline Tipo do detector & $\mathrm{UFPA}$, \\
$\begin{array}{l}\text { Faixa espectral } \\
\text { Tamanho do pixel }\end{array}$ & $7,5-13 \mu \mathrm{m}$ \\
Medição & $25 \mu \mathrm{m}$ \\
\hline Faixa de temperatura do objeto & $0 \ldots .250^{\circ} \mathrm{C}$ (utilizada) \\
Exatidão & $\pm 2^{\circ} \mathrm{C}$ ou $\pm 2 \%$ \\
\hline
\end{tabular}

O sensor utilizado foi previamente caracterizado em laboratório quanto às suas capacidades radiométricas, espectrais e espaciais, conforme metodologia descrita em [10] e os resultados apresentados em [11].

\subsection{Veículo aéreo não tripulado e Alvos de Referência}

A aeronave utilizada para obter as imagens foi um multirotor do tipo quadricóptero. Esta foi montada com uma controladora de voo Ardupilot, que é uma plataforma de código aberto para aeronaves pequenas, integrada com um sistema GNSS para posicionamento

Com o intuito de identificar nas imagens termais os pontos de controle e verificação em solo, foram utilizados 4 corner reflectors e 11 placas de alumínio, material de baixa emissividade, como alvos de referência. 


\subsection{Ortomosaico de referência e Coleta de dados}

Para a realização da avaliação posicional do ortomosaico gerados a partir da coleta de dados termais pelo VANT, pontos de verificação foram extraídos de um ortomosaico de referência. Estes produtos foram gerados por [12]. A obtenção das imagens com VANT foi realizada numa área de aproximadamente $10.000 \mathrm{~m}^{2}$. O tamanho do GSD-Ground Sample Distance planejado foi de $10 \mathrm{~cm}$. O planejamento de voo foi executado no software Mission Planner 1.3.39. Antes da realização do voo, pontos em campo foram sinalizados na área a ser sobrevoada. Esta sinalização foi realizada utilizando corner refletores e placas de alumínio. O objetivo de sinalizar os pontos termo-identificáveis foi proporcionar a coleta de coordenadas precisas nas imagens obtidas para posterior comparação com as coordenadas dos Gorund Control Points $(G C P)$ obtidos por GNSS em campo e pontos de verificação coletados dos produtos cartográficos de referência.

\subsection{Geração dos produtos e Avaliação da exatidão posicional}

Os dados coletados pelo sensor a bordo do VANT são registrados em matrizes numéricas de valores de temperatura em graus Celsius. Para processar esses dados no software de processamento dos dados VANT foi necessário realizar a sua conversão para o formato imagem (TIFF), procedimento realizado no software Matlab R2014b. Também houve a associação de cada imagem à sua coordenada correspondente, obtida pelo sistema GNSS a bordo do VANT. Essa associação foi realizada com base no horário de obtenção de cada um destes dados, sendo que para isso utilizou-se o software GeoSetter. Considerando que os dados foram pré-processados, eles foram inseridos no software Pix4D Mapper Pro (versão trial), tendo sido gerados de modo automático os ortomosaicos e modelos de superfície. Esse processamento foi realizado utilizando 4 pontos de apoio.

Após a geração dos ortomosaicos a partir das imagens termais obtidas durante o sobrevoo com VANT, procedeu-se a análise de exatidão posicional dos produtos gerados. Assim, a exatidão do ortomosaico foi verificada com base nos 25 pontos de verificação extraídos dos produtos de referência de acordo com o PEC-PCD usando um modelo matemático para análise estatística.

O método escolhido para avaliar os produtos de acordo com PEC-PCD, combinado com os conceitos apresentados pela Sociedade Americana de Fotogrametria e Sensoriamento Remoto - ASPRS (2014) [13]. (Figura 1)

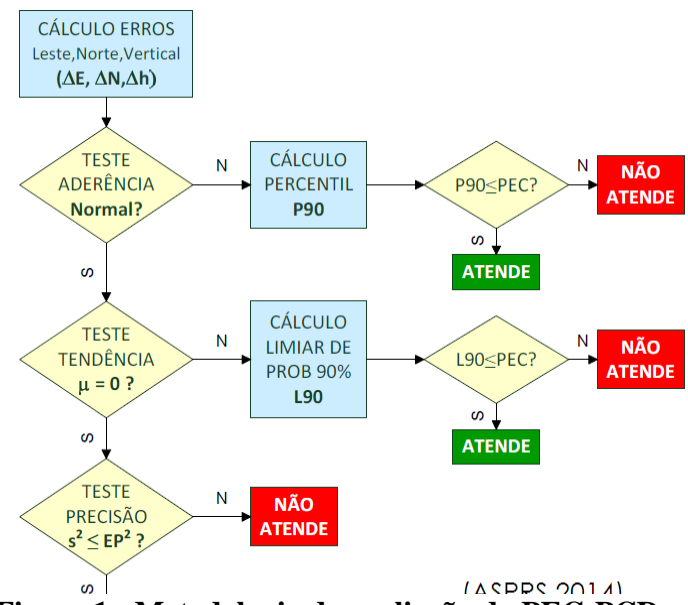

Figura 1 - Metodologia de avaliação do PEC-PCD

Portanto, para uma determinada escala, a imagem será classificada com a classe A, B, C, D ou 'nula' em função dos resultados obtidos nos testes de precisão.

\section{RESULTADOS}

Das 71 imagens coletadas, 64 foram utilizadas para a geração dos ortomosaicos. As imagens das bordas do bloco fotogramétrico com distorção muito elevada foram descartadas. Tais imagens, por causa da inclinação do VANT, na entrada e saída das faixas, foram tomadas fora da verticalidade ideal, gerando assim as distorções observadas e comentadas.
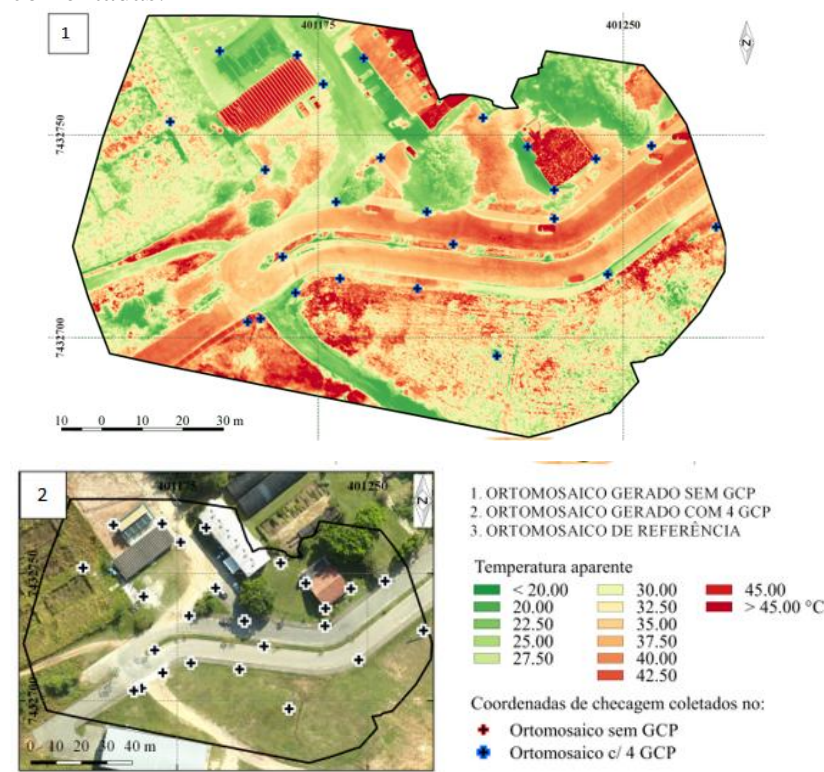

Figura 2. Ortomosaico termal (1) e de referência (2)

O processamento fotogramétrico foi realizado utilizando 4 GCPs distribuídos na área de estudo. O GSD obtido nos ortomosaicos foi $9,54 \mathrm{~cm}$. Após a geração do ortomosaicos, foram coletadas as coordenadas dos pontos de verificação em cada um através de um software específico com zoom da proporção 1:1. O ortomosaico termal gerado, bem como a posição das coordenadas neles coletadas e a ortofoto de referência estão representados na (Figura 2).

\subsection{Ortomosaico com GCPs (Análise qualitativa)}

No ortomosaico foram utilizados 4 GCPs (Tabela 2) coletados em campo com GNSS em seu processamento e 21 pontos de checagem que cujas coordenadas foram coletadas no ortomosaico de referência e medidas no ortomosaico a ser avaliado (E, N) e no modelo de elevação gerado $(\mathrm{H})$. Assim, os erros Leste $(\Delta \mathrm{E})$, Norte $(\Delta \mathrm{N})$, Planimétrico $(\Delta \mathrm{P})$ e Vertical $(\Delta \mathrm{H})$ oriundos do ortomosaico gerado utilizando 4 pontos de controle foram calculados e utilizados para avaliá-lo.

Tabela 2 - GCPs utilizados no ortomosaico (em metros)

\begin{tabular}{ccccccc}
\hline ID & $\mathbf{E}$ & $\mathbf{N}$ & $\mathbf{H}$ & $\boldsymbol{\Delta E}$ & $\mathbf{\Delta N}$ & $\Delta \mathbf{H}$ \\
\hline 5 & 401219.050 & 7432696.755 & 565.203 & 0.004 & 0.004 & 0.011 \\
6 & 401169.421 & 7432710.940 & 565.853 & 0.004 & 0.004 & 0.010 \\
10 & 401176.454 & 7432762.490 & 566.547 & 0.004 & 0.003 & 0.008 \\
42 & 401243.426 & 7432744.060 & 569.224 & 0.004 & 0.003 & 0.010 \\
\hline
\end{tabular}

A média dos erros planimétricos observada foi $0,51 \mathrm{~m}$ sendo a componente norte aquela que mais contribuiu para o distanciamento planimétrico dos pontos apresentando - $-0,17 \mathrm{~m}$ de deslocamento neste sentido. A média dos erros do sentindo leste foi $-0,07 \mathrm{~m}$, inferior ao tamanho do GSD do ortomosaico que foi $0,09 \mathrm{~m}$. O erro planimétrico máximo observado foi $1,70 \mathrm{~m}$. Na verificação a dispersão dos erros planimétricos do ortomosaico com GCPs. Dos 21 pontos observados, 15 ficaram dentro do RMS planimétrico de $0,65 \mathrm{~m}$. Este gráfico também permite observar que nesse ortomosaico os erros apresentam-se mais agrupados e próximos ao centro, o que 
demonstra maior precisão e exatidão em relação ao ortomosaico gerado sem pontos de controle.

$\mathrm{Na}$ verificação dos vetores de erros planimétricos. Observa-se que a maior parte dos erros planimétricos fo minimizado com a adoção de pontos de controle para o processamento. Essa melhoria foi observada em menor intensidade nos pontos localizados nas extremidades do ortomosaico.

A maior parte dos erros $(\sim 76 \%)$ foi negativa, indicando que o modelo gerado com quatro pontos de controle subestimou os valores de altitude. Os maiores erros observados foram de $-7,47 \mathrm{~m},-6,73 \mathrm{~m}$ e $-3,02 \mathrm{~m}$. A média dos erros altimétricos foi de $1,43 \mathrm{~m}$. Dos 21 pontos observados, alguns foram escolhidos para a melhor representação dos desvios planimétricos observados, sendo que em dois deles os desvios foram mais bem resolvidos com a adoção de pontos de controle, e dois onde o erro, apesar de minimizado, ainda foi significativo para a avaliação da precisão do ortomosaico.

Um dos pontos analisados foi o P07 (Figura 3). Os erros observados neste ponto nos ortomosaicos sem e com GCPs, respectivamente, foram de $2,42 \mathrm{~m} \mathrm{e} 0,11 \mathrm{~m}$. Este ponto apresentou o menor erro no ortomosaico com GCPs. Tal melhoria pode ser atribuída a sua proximidade posicional a um dos pontos de controle utilizados no processamento (P06), o que permite ressaltar a importância de um maior número de GCPs bem distribuídos na área para a geração de ortomosaicos.

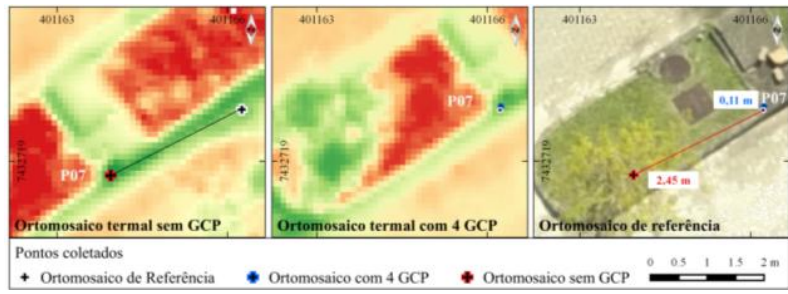

Figura 3. Desvios observados no ponto de verificação P07

A análise descritiva apresentada permitiu identificar a melhoria na qualidade posicional do ortomosaico quando gerado utilizando 4 GCPs. Entretanto demonstra que um número maior de pontos de apoio poderia minimizar erros que permaneceram elevados mesmo considerando 4 GCPs. Observou-se também um maior deslocamento nos pontos posicionados na extremidade do ortomosaico e em áreas com menor sobreposição.

Para uma avaliação mais detalhada sobre a qualidade do ortomosaico gerados será apresentada a seguir a análise estatística de exatidão posicional realizada sobre os produtos oriundos do levantamento de dados termais com VANT, bem como a sua classificação em relação ao PEC-PCD.

\subsection{Ortomosaico com GCPs (Análise Estatística} classificação quanto ao PEC)

Os erros observados para os 21 pontos de verificação do ortomosaico gerado utilizando 4 GCPs também foram analisados de acordo com a metodologia apresentada na Figura 6. O primeiro teste foi quanto à normalidade das amostras, utilizando o teste Kolmogorov-Smirnov-ks(Tabela $3)$.

Os erros nos sentidos leste, norte e vertical apresentaram p-valor 0,0121, 0,0044 e 1,5909-04, respectivamente, e assim, ao nível de significância de 5\%, rejeitam a hipótese nula de que as amostras possuem distribuição normal e, portanto tiveram sua exatidão analisada a partir da comparação dos percentis em relação ao PEC-PCD.

As amostras foram ainda testadas quanto à tendência através do teste $t$-Student com a hipótese nula de média igual 0 e a um nível de significância de $1 \%$. Todas as amostras apresentaram um p-valor maior que 0,05 demonstrando que os erros estão livres de tendência.
Tabela 3. Resultado do $k s$ dos erros com $G C P s$

\begin{tabular}{cc}
\hline Nível de confiança & $95 \%$ \\
\hline Nível de significância & $5 \%$ \\
Amostra & 21 \\
Graus de Liberdade & 20 \\
Ks tabelado & 0,2813 \\
\hline Ks calculado E & 0,3380 \\
Têm distribuição normal? & Não \\
\hline p-valor & 0,0121 \\
\hline Ks calculado N & 0,3699 \\
\hline Têm distribuição normal? & Não \\
p-valor & 0,0044 \\
\hline Ks calculado H & 0,4567 \\
Têm distribuição normal? & Não \\
p-valor & $1,5909 \mathrm{E}-04$ \\
\hline
\end{tabular}

Segundo a metodologia de avaliação, as amostras que não apresentam distribuição normal, devem ser analisadas a partir da comparação por percentil. Assim, verificou-se se $90 \%$ dos erros observados são inferiores aos valores determinado pelo PEC-PCD para uma dada escala e classe. Os valores dos percentis de $90 \%$ obtidos foram: $1,1323 \mathrm{~m}$ para o erro planimétrico, 0,8996 m para o erro no sentido norte, $0,7850 \mathrm{~m}$ para o erro no sentido leste e $4,5008 \mathrm{~m}$ para o erro no sentido vertical.

Os percentis calculados foram comparados com os valores do PEC-PCD estabelecidos para cada classe e escala nos sentidos norte e leste, planimétrico e altimétrico.

Dessa forma, constatou-se que analisado pelos erros planimétricos, ao nível de significância de $\alpha=1 \%$, o ortomosaico com 4 GCPs obteve a classe D a partir de 1:1.000, B a partir de 1:2.000 e A a partir da escala 1:5.000 (Tabela 4). As mesmas classes nas referidas escalas foram atribuídas ao ortomosaico quando avaliado pelos erros no sentido norte (Tabela 4).

No sentido leste, o ortomosaico obteve a classe $\mathrm{C}$ a partir da escala 1:1.000, B a partir da escala 1:2.000 e A a partir da escala 1:5.000 (Tabela 4). Na vertente altimétrica este ortomosaico obteve classe B a partir de 25.000 e A a partir de 1:50.000 (Tabela 4). Nas demais escalas o produto cartográfico foi rejeitado em relação à exatidão dos valores.

Para efeito de comparação a tabela 17 foi organizada com um resumo das classes obtidas para cada escala nos dois ortomosaicos.

Tabela 4. Classificação dos ortomosaicos de acordo o PEC

\begin{tabular}{|c|c|c|c|c|c|c|c|c|c|}
\hline Escala (x100) & $\stackrel{n}{n}$ & $\stackrel{\text { ㅇ․ }}{\ddot{ت}}$ & ن. & مِ & $\begin{array}{l}\stackrel{8}{0} \\
\ddot{-}\end{array}$ & $\begin{array}{l}\stackrel{\circ}{n} \\
\stackrel{n}{-}\end{array}$ & 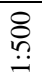 & $\begin{array}{l}\stackrel{8}{\circ} \\
\stackrel{-}{*}\end{array}$ & $\begin{array}{l}\stackrel{8}{0} \\
\stackrel{n}{-}\end{array}$ \\
\hline \multicolumn{10}{|c|}{ COM 4 GCPs (Ground Control Points) } \\
\hline Leste & - & $\overline{\bar{C}}$ & $\overline{\overline{\mathrm{B}}}$ & $\overline{\mathrm{A}}$ & $\overline{\overline{A \mathrm{~A}}}$ & $\overline{\mathrm{A}}$ & $\overline{\mathrm{A}}$ & $\overline{\mathrm{A}}$ & $\overline{4}$ \\
\hline Norte & - & $\mathrm{D}$ & B & $\mathrm{A}$ & $\bar{A}$ & $\mathrm{~A}$ & $\mathrm{~A}$ & $\mathrm{~A}$ & $\mathrm{~A}$ \\
\hline Planimétrico & - & $\mathrm{D}$ & B & $\mathrm{A}$ & $\mathrm{A}$ & $\mathrm{A}$ & $\bar{A}$ & $\mathrm{~A}$ & $\mathrm{~A}$ \\
\hline Vertical & - & - & - & - & - & B & A & $\mathrm{A}$ & A \\
\hline
\end{tabular}

Como resultado final das avaliações realizadas conclui-se que o ortomosaico gerado sem pontos de controle demonstrou exatidão planimétrica a partir da escala 1:5.000 e altimétrica a partir de 1:50.000. O ortomosaico processado com 4 pontos de controle, apresentou exatidão altimétrica a partir de 1:25.000 e planimétrica a partir da escala 1:1.000. No entanto, como mencionado na seção 3.1, o ortomosaico utilizado com referência possui exatidão planimétrica a partir da escala 1:1.000 e, portanto, só pode ser utilizado como referência para a avaliação de produtos a partir da escala 1:3.000, já que a precisão dos pontos de verificação dever ser de pelo menos $1 / 3$ da precisão avaliada. Assim, quanto ao PEC-PCD, é considerada a exatidão do ortomosaico com 4 GCPs a partir da escala 1:3.000 com classe B e classe A partir da escala $1: 5.000$.

\section{CONSIDERAÇÕES FINAIS}


Na avaliação da exatidão posicional dos produtos gerados através dos dados termais levantados por VANT nesta pesquisa, constatou-se que o uso de sensores que operam na faixa espectral do infravermelho termal é eficaz na obtenção de dados para a geração de ortomosaicos, contudo o uso de pontos de controle é indispensável para se obter uma boa qualidade do produto cartográfico resultante. Os resultados da avaliação dos ortomosaicos com o uso de pontos de controle no seu processamento, demonstram que a sua utilização melhora consideravelmente a exatidão posicional do produto, permitindo que este poderá ser utilizado em aplicações que exijam maior precisão.

Observou-se que com a adoção de apenas 4 GCPs houve uma melhoria significativa no posicionamento e, com um maior número de GCPs bem distribuídos na área de estudo a exatidão alcançada tende a ser maior. Sobre a distribuição dos GCPs recomenda-se que haja pontos localizados nas extremidades da área a fim de evitar deslocamentos maiores nestas regiões. $\mathrm{O}$ uso da metodologia de avaliação posicional para a avaliação dos produtos gerados por dados termais se mostrou válida, desde que alvos compostos por materiais de baixa emissividade sejam utilizados para a identificação dos pontos de checagem na imagem termal. Vale ressaltar que o tamanho dos alvos utilizados, para experimentos como o apresentado, precisa ser considerado em relação ao tamanho do $G S D$ planejado para a missão. Recomenda-se que o alvo utilizado tenha pelo menos 3 vezes o tamanho do GSD. Se o ponto de checagem considerado estiver no limite de alvos com emissividades muito discrepantes, o uso de tais alvos pode ser dispensável.

Se comparadas, as classificações de exatidão planimétrica do ortomosaico de referência (A - 1:1.000) e aquela obtida pelo ortomosaico gerado com GCPs (A-1:5.000), são proporcionais considerando-se seus respectivos tamanhos de $G S D(2,10$ e $9,54 \mathrm{~cm})$. Isso ocorre porque se verifica que o ortomosaico termal que possui um GSD aproximadamente 5 vezes maior, obteve a classe A quanto ao PEC-PCD para uma escala 5 vezes maior. A exatidão posicional do ortomosaico gerado com dados termais mostrou-se comparável a exatidão de um ortomosaico gerado com dados obtidos na faixa do visível.

$\mathrm{Na}$ área analisada, diferenças significativas de temperatura radiante puderam ser observadas em alvos que visivelmente pareciam iguais, por exemplo na vegetação, telhados e em áreas sombreadas. Assim, desde que vinculado a utilização de pontos de apoio para o processamento e consideradas as incertezas envolvidas no seu processo de obtenção, constata-se a viabilidade do uso de dados termais obtidos por VANT como uma alternativa eficaz para o imageamento de áreas com características não aparentes na faixa do visível, ou em períodos noturnos.

\section{REFERÊNCIAS}

[1] Jensen, J. R. Sensoriamento remoto do ambiente. 2. ed. São José dos Campos: Parêntese, 2009.

[2] Salamí, E.; Barrado, C.; Pastor, E. UAV Flight Experiments Applied to the Remote Sensing of Vegetated Areas. Remote Sensing. v.6. 2014.

[3] Smigaj, M; Gaulton, R.; Barr, S. L.; Suárez, J. C. UavBorne Thermal Imaging for Forest Health Monitoring: Detection of Disease-Induced Canopy Temperature Increase. In: The Int. Arch. Photog., Rem.Sens. \& Spatial Information Sciences, Volume XL-3/W3. 2015.

[4] Berni, J.;Zarco-Tejada, P.J.; Suarez, L.; Fereres, E. Thermal and Narrowband Multispectral Remote Sensing for Vegetation Monitoring From an Unmanned Aerial Vehicle IEEE Trans. Geoscience \& Remote Sensing. v. 47. n. 3. 2009.
[5] Hoffmann, H.; Nieto, H.; Jensen, R.; Guzinski, R.; ZarcoTejada, P. J.; Friborg, T. Estimating evapotranspiration with thermal UAV data and two source energy balance models. Hydrol. \& Earth System Sciences Discussions. v.12, p. 7469 7502. 2015.

[6] Kontoes, C.; Keramitsoglou, I.; Sifakis, N.; Konstantinidis, P. Sithon: An Airborne Fire Detection System Compliant with Operational Tactical Requirements. Sensors (Basel). 2009; 9(2): 1204-1220.

[7] Matikainen, L.; Lehtomäkia, M.; Ahokasa, E.; Hyyppäa, J.; Karjalainena, M.; Jaakkolaa, A.; Kukkoa, A.; Heinonenb, T. Remote sensing methods for power line corridor surveys. ISPRS J. of Photog. \& Rem. Sensing. v. 119, 2016, (p10-31).

[8] Lega, M.; Napoli, R. M. A. Aerial infrared thermography in the surface waters contamination monitoring. In: Proceedings of the 11th International Conference on Environmental Science and Technology. Chania, Greece. 2009

[9] OPTRIS, GMBH. Operator's Manual: OPTRIS PI LightWeight Kit. CD-ROM. 2016

[10] Kux, H. J. H.; Oliveira, L. T.; Castro, R. M. Análise das capacidades e limitações radiométricas, espectrais e espaciais de dois sistemas sensores termais em laboratório. In: XVIII SBSR, Santos, 2017. Anais. 2017, p.4575-4582.

[11] Oliveira, L. T.; Castro, R. M.; Kux, H. J. H. Metodologia para a caracterização de sistemas sensores termais. In: XVIII SSBSR, Santos, 2017. Anais. 2017, p.5788-5795.

[12] Nogueira, F. C.; Roberto, L.; Körting, T. S.; Shiguemori, E. H. Accuracy analysis of orthomosaic and DSM produced from sensor aboard UAV. In: XVIII SSBSR, Santos, 2017. Anais. 2017, p.5515-5521.

[13] ASPRS. Positional Accuracy Standards for Digital Geospatial Data, 1st Ed, V.1, ASPRS 2014. Available in: <https://www.asprs.org/publications-other/manualofphotogrammetry-errata.html>. Access: 16.jun.2016.

[14] Kontoes, C.; Keramitsoglou, I.; Sifakis, N.; Konstantinidis, P. Sithon: An Airborne Fire Detection System Compliant with Operational Tactical Requirements. Sensors (Basel). 2009; 9(2): 1204-1220

.[15] McGlone, J.C; Lee, G.Y.G. Manual of Photogrammetry, Sixth Edition. ASPRS, 2013. ISBN 1-57083-099-1.

[16] M; Kosmatka, J.; Ferrara, C.; Russo, F.; Napoli, R. M. A.; Persechino, G. Using Advanced Aerial Platforms and Infrared Thermography to Track Environmental Contamination. Environmental Forensics. v.13. n. 4. 2012

[17] Merchant, D.C. Spatial Accuracy Standards for Large Scale Line Maps. Technical Papers of Am.Congress Surv. \& Mapping, Denver-CO, USA, 1982. p. 222-231.

[18] Warren, M. S. Desagregação espacial de estimativas de evapotranspiração real obtidas a partir do sensor modis. Rev. Bras. Meteorologia, v. 28, n. 2, p. 153-162, jun. 2013.

[19] Watts, A.C., Ambrosia, V.G., Hinkley, E.A. Unmanned Aircraft Systems in Remote Sensing and Scientific Research: Classification and Considerations of Use. Remote Sensing. 4(6), 1671 - 1692. 2012

[20] Wolf, P.R.; Dewitt, B.A.; Wilkinson, B.E. Elements of Photogrammetry with Aplications in GIS, Fourth Edition. Singapore, Mc Graw-Hill, 2014. 676p. ISBN 978-0-07176112-3. 